\title{
Alterations of oral functions and dental malocclusions in adolescents: a cross-sectional population-based study
}

\author{
Alterações das funções orais e má oclusão em adolescentes: \\ um estudo transversal de base populacional
}

Heloiza Viana Freitas (https://orcid.org/0000-0003-0243-098X) ${ }^{1}$

Cláudia Maria Coelho Alves (https://orcid.org/0000-0003-4705-4914) ${ }^{1}$

Luciana Freitas Gomes e Silva (https://orcid.org/0000-0001-9727-5112) ${ }^{2}$

Alex Luiz Pozzobon Pereira (https://orcid.org/0000-0001-5648-9878) ${ }^{1}$

Fernando Neves Hugo (https://orcid.org/0000-0003-2222-7719) ${ }^{3}$

Erika Barbara Abreu Fonseca Thomaz (https://orcid.org/0000-0003-4156-4067) ${ }^{1}$

\footnotetext{
${ }^{1}$ Programa de PósGraduação em Odontologia, Universidade Federal do Maranhão. Av. Dos Portugueses 1966, Bacanga. 65080-805 São Luís MA Brasil.

lucianafgs@gmail.com

${ }^{2}$ Departamento de Odontologia, Universidade CEUMA. São Luís MA Brasil.

${ }^{3}$ Departamento de Odontologia Preventiva e Social, Universidade Federal do Rio Grande do Sul. Porto Alegre RS Brasil.
}

\begin{abstract}
This article aims to assess whether alterations of oral functions (AOF) are associated with malocclusion (MO)'s type and severity. Cross-sectional study of a representative sample of 332 adolescents aged 12 years in São LuisMA, Northeastern Brazil. MO criteria included Angle's classification, Dental Aesthetic Index, and other morphological problems. The AOF were evaluated by breathing, phonation, chewing, and swallowing. Odds ratios (OR) and 95\% confidence intervals $(95 \% \mathrm{CI})$ were calculated using logistic and multinomial regression analyses $(\alpha=5 \%)$. Mouth breathing was associated with defined $(O R=3.84 ; 95 \% C I=1.45-10.12)$, disabling (OR=4.34; 95\%CI=1.99-9.49), and class III $(O R=4.15 ; 95 \% C I=1.19-14.54)$ MO. Phonation problems were associated with defined $(O R=2.01 ; 95 \% C I=1.02-4.39)$, disabling $(\mathrm{OR}=3.04 ; 95 \% \mathrm{CI}=1.55-5.96)$, and Class II $(O R=2.02 ; \quad 95 \% C I=1.28-3.18) \quad M O$. Chewing disorders were associated with posterior crossbite (PCB) $(\mathrm{OR}=2.32$; $95 \% \mathrm{CI}=1.12-4.82)$. Swallowing disorders were associated with Class III MO $(O R=5.66 ; 95 \% C I=1.35-23.71), P C B(O R=6.13$; 95\%CI $2.76-13.62)$, and posterior open bite $(\mathrm{OR}=4.53$; 95\%CI=1.72-8.92). Breathing and phonation alterations are associated with $M O$ in anterior arch segments, while chewing and swallowing disorders, in the posterior segments.
\end{abstract}

Key words Malocclusion, Mouth Breathing, Phonation, Swallowing
Resumo $O$ objetivo deste artigo é avaliar se alterações das funções orais (AFO) estão associadas ao tipo e gravidade da maloclusão (MO). Estudo transversal com amostra representativa de 332 adolescentes de 12 anos em São Luís-MA, Nordeste do Brasil. Critérios de MO incluiram Classificação de Angle, Índice de Estética Dental e outros problemas morfológicos. As AFO foram avaliadas por respiração, fonação, mastigação $e$ deglutição. Odds ratios (OR) e intervalos de confiança de 95\% (IC95\%) foram calculados em análises de regressão logística e multinomial $(\alpha=5 \%)$. Respiração oral foi associada com $M O$ definida $(O R=3,84 ; \quad I C 95 \%=1,45-10,12)$, incapacitante $(\mathrm{OR}=4,34 ; \quad I C 95 \%=1,99-9,49) \quad e$ classe III $(O R=4,15$; IC95\%=1,19-14,54). Problemas de fonação foram associados às MO definidas (OR=2,01; IC95\%=1,02-4,39), incapacitantes $(O R=3,04 ; \quad I C 95 \%=1,55-5,96)$ e Classe II $(\mathrm{OR}=2,02$; IC95\%=1,28-3,18). Alterações na mastigação foram associadas à mordida cruzada posterior (MCP) $(\mathrm{OR}=2,32$; IC95\%=1,12-4,82). Deglutição atípica foi associada à MO Classe III $(O R=5,66 ;$ IC95\%=1,35-23,71), $M C P(O R=6,13$; IC95\% $=2,76-13,62)$ e mordida aberta posterior $(O R=4,53 ;$ IC95\%=1,72-8,92). Alterações de respiração e fonação estão associadas às $M O$ nos segmentos anteriores do arco, enquanto as de mastigação e deglutição, nos segmentos posteriores.

Palavras-chave Má oclusão, Respiração Bucal, Fonação, Deglutição 


\section{Introduction}

Dental malocclusion is a major oral health problem ${ }^{1,2}$, given its high magnitude and aesthetic impact $^{3,4}$, possibly affecting psychosocial ${ }^{5}$ and quality of life $^{6}$ aspects. Its prevalence varies globally. While $83.3 \%{ }^{7}$ is recorded in India, in Brazil, $19.8 \%$ of 12 -year-olds have very severe or disabling malocclusions, as per data from the latest national oral health survey ${ }^{1}$.

Many criteria are available to diagnose malocclusions. DAI was chosen mainly for providing objective measures of different occlusal problems and enabling prioritization of treatment needs ${ }^{1,2}$. Thus, the DAI expresses much more the shape of the anterior region of the dental arch than the orofacial function ${ }^{8}$. Although there are indications that function interferes in the form of biological structures ${ }^{9-15}$, the relationship between functional alterations of the stomatognathic system and malocclusions is not yet fully understood. Some studies have suggested an association between changes in breathing ${ }^{9-11,14}$, speech adaptations ${ }^{12,13}$, and swallowing ${ }^{13,15}$ with malocclusions. However, other authors found no association between functional alterations and malocclusions ${ }^{16-18}$.

There is no consensus on the association of functional alterations with malocclusions, nor whether they influence malocclusion's type and severity. Studies generally evaluate malocclusion through stringent criteria and extensive age groups ${ }^{4,15}$. Also, they do not account for the sample design ${ }^{3,13}$, do not evaluate changes in the chewing function ${ }^{13,15}$, nor consider the possibility of confounding bias in statistical analyses ${ }^{3,13}$. This study has a large sample. It considered the study design and confounding biases, and performed chewing evaluations.

Therefore, the study aimed to assess the association between functional alterations of the stomatognathic system and malocclusion. The research hypothesizes that functional alterations can cause muscle imbalance, with repercussions in the modeling/remodeling of craniofacial bones, as well as in teeth position in the dental arches, harming dentofacial harmony and balance, thus associating with high prevalence and severity of malocclusions.

\section{Methods}

\section{Study Area and Design}

This study followed the STROBE (Strengthening the Reporting of Observational Studies in Epidemiology) guidelines ${ }^{19}$. This is a cross-sectional population-based study held in São Luís, Brazil. São Luís is located on an island on the northern coast of the state of Maranhão, Northeastern Brazil. The human development index (HDI) was 0.768 in the last Brazilian census, in 2010, ranking first in Maranhão and $249^{\text {th }}$ in Brazil, and had 1,014,837 inhabitants. At the time, the monthly per capita household income was US\$ 434.54, with a Gini index of 0.627 and illiteracy rate of $4.6 \%{ }^{20}$.

\section{Sampling design}

Students aged 12 years of both genders enrolled in public and private elementary schools were the reference population. The sample design was two-stage clustered and stratified by school type (public or private). Schools (primary sampling units) and students (secondary sampling units) were randomly selected. Lists of schools available at the Ministry of Education, Municipal Secretariat of Education of São Luís-MA, and schools themselves were used for the draw. The sample size at each school was proportional to the number of students in the school.

We used the Epi-Info software, version 6.0, to estimate the sample size. A sample of 180 adolescents would have a $95 \%$ power to detect significant prevalence ratios (PR) of $2.31^{21}$, considering the prevalence of $19.8 \%$ of malocclusion among unexposed subjects ${ }^{1}$, a $95 \%$ confidence level, and 1:1 ratio between unexposed and exposed subjects. We included an additional sample of 50\% to supply possible losses, resulting in a sample of 270 adolescents. Besides, considering the effect of the complex sample design (deff) of $1.2^{22,23}$, we added other 54 adolescents, reaching a minimal sample of 324 adolescents (162 unexposed and 162 exposed to at least one of the oral functional alterations investigated in this study).

The data collection period was March to September 2013. Inclusion criteria were 12 years of age and being enrolled in one of the elementary schools of São Luís. Students with syndromes, current or previous history of orthodontic-orthopedic treatment or myofunctional and articulatory therapy declared by parents or students, and those who have lost the first permanent molars were excluded. 
the Kappa test and the intraclass correlation test, accepting minimum values of 0.7 . The intraclass repeatability ranged from 0.88 to 0.91 for clinical dental examinations and DAI. The inter-examiner repeatability for clinical dental examinations and DAI among the two examiners ranged from 0.79 to 0.81 .

\section{Pilot study}

A pilot was performed considering all the variables involved in the study. The pilot study was carried out in a school with 32 adolescents to test the administration of questionnaires and clinical examination of schoolchildren, simulating real data collection situations. Those who participated in the pilot study were not included in the final sample. Adolescents participating in the pilot study received and answered the questionnaire, which was deemed appropriate, and no changes were necessary.

\section{Clinical data collection}

Clinical data were collected at school under a natural light source. Dental examinations were performed as per recommendations of the $\mathrm{WHO}^{2}$. An endodontic millimeter ruler, adapted silicone stop, and orthodontic wire were used for measurements ${ }^{24}$ in the orthodontic examination.

Malocclusions were the outcomes of interest, which were evaluated through different criteria: Angle classification ${ }^{25}$; $\mathrm{DAI}^{26}$; and morphological changes not included in DAI - posterior open bite, posterior crossbite, and overbite ${ }^{27}$.

Students were categorized as Class I, Class II (subdivision 1 and 2), and Class $\mathrm{III}^{3}$ in Angle's classification. DAI was adopted to evaluate the presence and severity of malocclusion and the normative need for orthodontic treatment $(\mathrm{NOT})^{1,18}$. It consists of 10 occlusal components divided into three dimensions: teething problems, occlusion, and space ${ }^{26,27}$. According to DAI, we classified student's malocclusion as normal/ mild malocclusion (DAI $\leq 25$; no need for treatment); defined malocclusion (DAI=26-30, elective need); severe malocclusion (DAI $=31-35$, highly desirable need); or disabling malocclusion (DAI $\geq 36$, required need). Given that DAI does not mention morphological disorders such as crossbite, posterior open bite, and overbite ${ }^{27}$, the evaluation of these items was included in the clinical examination.

Functional alterations of the stomatognathic system (breathing, phonation, chewing, and swallowing) were the main independent variables. The assessment followed the recommendations of some authors ${ }^{13,18,28}$.

In the evaluation of the respiratory function, three criteria were observed. The first criterion concerned the lip seal without voluntary muscle contraction, whose presence is a sign of normality. The second criterion was related to the time in which the child could breathe with sealed lips, which was considered normal when the child could breathe this way for at least one minute. The third criterion consisted of placing a small mirror under the child's nostrils, who was requested to breathe normally. Mirror haze was considered a sign of normality. The presence of mouth breathing was characterized when at least one of these criteria was changed ${ }^{18}$.

Adolescents with mandibular deviation, anterior or lateral tongue projection in the moment of articulation of phonemes or pronunciation of phonemes with sigmatism (vicious repetition of the " $\mathrm{s}$ " and other sibilants) were considered carriers of phonation problems ${ }^{13}$.

About $20 \mathrm{~g}$ of bread was given to adolescents to identify chewing problems, evaluating the following criteria: labial sealing, accumulation of food in the oral vestibule, chewing side, and exaggerated participation of perioral muscles. Adolescents showing a change in one or more criteria were considered with chewing problem ${ }^{29}$.

Swallowing was assessed by asking the child to put a small amount of mineral water (about $30 \mathrm{ml}$ ) in the mouth and not swallow immediately. Then, using two wooden disposable spatulas to separate lips, the child was asked to swallow the water normally. This process was repeated twice. The swallowing pattern was considered atypical in the presence of lingual interposition between arches or when the tongue atypically pressed some front teeth ${ }^{13}$.

\section{Non-clinical data collection}

The following covariates were also considered in the study: 1) sociodemographic data: gender (male or female); school type (public or private), economy class as per the Brazilian Association of Research Companies' (ABEP, acro- 
nym in Portuguese) criteria $(\mathrm{A} / \mathrm{B}, \mathrm{C}$ or $\mathrm{D} / \mathrm{E})$ and self-reported skin color, according to Brazilian Institute of Geography and Statistics (IBGE, acronym in Portuguese) (white, black or other, including brown, yellow and red), school failure history (yes or no); 2) behavioral data: current or previous history of deleterious oral habits (pacifier/finger sucking, biting lips or cheeks, biting objects, nail-biting, gnashing teeth, sleeping with hands under the face or keeping hand under the chin and lingual interposition). The demographic and behavioral data were collected through a questionnaire.

\section{Statistical analysis}

The questionnaires were coded and revised in search of inconsistencies. Analyses were performed using Stata software, version 11.0 (Stata Corp., College Station, Texas, USA). Frequencies between groups were compared by Fisher's exact, chi-square, and chi-square (linear trend) tests. A significance level of 5\% was adopted for rejecting null hypotheses in all analyses. Logistic and multinomial regression analyses with stepwise modeling strategy were performed, calculating the non-adjusted and adjusted odds ratio (OR). Covariates with $\mathrm{P}<0.2$ in non-adjusted associations between malocclusion and covariates were considered potential confounders. Only covariates with $\mathrm{P}<0.10$ in the adjusted analyses remained in the final model ${ }^{30-32}$. All analyses considered the design effect in estimating standard errors and were weighted by the inverse of the selection probability. We tested multicollinearity using the Stata command_rmcoll.

\section{Ethical considerations}

The study was approved by the Research Ethics Committee of the University Hospital of the Federal University of Maranhão. Written informed consent was obtained from the State Department of Education and school principals. The parents/ guardians of the schoolchildren also signed a consent form after being informed about the research objectives, agreeing to the adolescents' participation in the research. We excluded students if their parents/guardians withdrew them from the study and if they declined to take part in the research.

\section{Results}

A total of 400 students, all aged 12 years old, were evaluated for their eligibility to participate in the study. We excluded 51 students: 17 were already receiving orthodontic treatment, and 34 had lost at least one first permanent molar. Of the 349 eligible students, 11 students refused to participate in the study and six students were not present in school at the second evaluation.

The sample consisted of eleven public and five private schools, totaling 332 students, with $82.7 \%$ public schools and $17.3 \%$ private schools. Most schoolchildren were male $(53.1 \%)$, brown (65.2\%), economy class C (59.7\%), and had no history of school failure (66.5\%).

Tables 1 and 2 show the malocclusions frequency distribution and study covariates, as per the occurrence of functional alterations. Differences were found in the malocclusion distribution, according to DAI ( $\mathrm{p}=0.002)$, among those who had or not breathing alterations. A difference was found in the distribution of malocclusion, as per DAI ( $p=0.048)$ and Angle $(\mathrm{p}=0.003)$, by type of phonation problems (Table 1 ). Table 2 showed that the prevalence of $\mathrm{MO}$ according to Angle and posterior crossbite varied among patients with chewing alterations $(p=0.012$ and $\mathrm{p}=0.031$ ) as well as among these with swallowing disorders $(\mathrm{p}=0.027$ and $\mathrm{p}<0.001)$.

In the univariate regression analysis, as per DAI, adolescents with breathing alterations were more likely to have defined and disabling malocclusions. There was a collinear relationship between the type of school and economic class, so the first one was excluded from the adjusted analysis. After adjusting for confounders, it was found that the likelihood of having defined $(\mathrm{OR}=3.84 ; 95 \% \mathrm{CI}=1.45-10.12)$ and disabling malocclusions $(\mathrm{OR}=4.34 ; 95 \% \mathrm{CI}=1.99$ 9.49) and Class III malocclusion ( $\mathrm{OR}=4.15$; $95 \% \mathrm{CI}=1.19-14.59)$ was higher among patients with breathing problems, compared to those with normal breathing (Table 3 ).

Association between phonation and disabling (DAI) and Class II malocclusion (Angle) was observed (Table 3). After adjustment of models, the likelihood of having defined malocclusion $(\mathrm{OR}=2.01 ; 95 \% \mathrm{CI}=1.02-4.39)$, disabling malocclusion $\quad(\mathrm{OR}=3.04 ; \quad 95 \% \mathrm{CI}=1.55-5.96)$ and Class II malocclusion of Angle $(\mathrm{OR}=2.02$; $95 \% \mathrm{CI}=1.28-3.18)$ was higher among patients with phonation problems when compared to those with normal phonation. The strength of associations was higher in disabling malocclusion. Although severe malocclusion was associated neither with breathing nor phonation alterations, the trend chi-square test was significant (Table 1).

There was an association between chewing disorders and posterior crossbite in both 
Table 1. Malocclusions and sociodemographic and behavioral characteristics of the sample, according to breathing and phonation alterations. São Luís, Brazil.

\begin{tabular}{|c|c|c|c|c|c|c|c|c|c|c|}
\hline \multirow{3}{*}{ Variables } & \multicolumn{4}{|c|}{ Breathing alterations } & \multirow{3}{*}{$\begin{array}{c}\text { P- } \\
\text { value }\end{array}$} & \multicolumn{4}{|c|}{ Phonation alterations } & \multirow{3}{*}{$\begin{array}{c}\text { P- } \\
\text { value }\end{array}$} \\
\hline & \multicolumn{2}{|c|}{ No } & \multicolumn{2}{|c|}{ Yes } & & \multicolumn{2}{|c|}{ No } & \multicolumn{2}{|c|}{ Yes } & \\
\hline & $\mathbf{n}$ & $\%$ & $\mathbf{n}$ & $\%$ & & $\mathbf{n}$ & $\%$ & $\mathbf{n}$ & $\%$ & \\
\hline Malocclusion (DAI) & & & & & $0.002^{1}$ & & & & & $0.048^{1}$ \\
\hline Normal/Mild & 81 & 84.1 & 16 & 15.9 & $0.007^{2}$ & 69 & 70.0 & 28 & 30.0 & $0.029^{2}$ \\
\hline Defined & 51 & 55.1 & 31 & 44.9 & & 47 & 51.9 & 35 & 48.1 & \\
\hline Severe & 60 & 77.5 & 19 & 22.5 & & 51 & 61.8 & 28 & 38.2 & \\
\hline Disabling & 43 & 54.2 & 31 & 45.8 & & 34 & 43.5 & 40 & 56.5 & \\
\hline Malocclusion (Angle) & & & & & $0.494^{3}$ & & & & & $0.003^{3}$ \\
\hline Normal & 10 & 85.3 & 2 & 14.7 & & 11 & 96.5 & 1 & 3.5 & \\
\hline Class I & 142 & 70.1 & 56 & 29.9 & & 130 & 63.3 & 98 & 36.7 & \\
\hline Class II & 72 & 72.5 & 31 & 27.5 & & 51 & 53.1 & 52 & 46.9 & \\
\hline Class III & 11 & 41.7 & 8 & 58.3 & & 9 & 30.5 & 10 & 69.5 & \\
\hline Posterior crossbite & & & & & $0.085^{1}$ & & & & & $0.280^{1}$ \\
\hline No & 201 & 73.1 & 76 & 26.9 & & 170 & 60.8 & 107 & 39.2 & \\
\hline Yes & 34 & 53.4 & 21 & 46.6 & & 31 & 47.9 & 24 & 52.1 & \\
\hline Posterior open bite & & & & & $0.384^{1}$ & & & & & $0.298^{1}$ \\
\hline No & 214 & 70.2 & 83 & 29.8 & & 184 & 59.8 & 113 & 40.2 & \\
\hline Yes & 21 & 65.0 & 14 & 35.0 & & 17 & 48.0 & 18 & 52.0 & \\
\hline Overbite & & & & & $0.165^{1}$ & & & & & $0.927^{1}$ \\
\hline No & 142 & 72.9 & 51 & 27.1 & & 118 & 58.7 & 75 & 41.3 & \\
\hline Yes & 93 & 63.7 & 46 & 36.3 & & 83 & 58.2 & 56 & 41.8 & \\
\hline Sex & & & & & $0.724^{1}$ & & & & & $0.147^{1}$ \\
\hline Female & 109 & 68.2 & 45 & 31.8 & & 92 & 52.3 & 62 & 47.7 & \\
\hline Male & 126 & 70.9 & 52 & 29.1 & & 109 & 64.0 & 69 & 36.0 & \\
\hline Race/color & & & & & $0.166^{1}$ & & & & & $0.082^{1}$ \\
\hline White & 65 & 79.5 & 16 & 20.5 & & 49 & 54.5 & 32 & 45.5 & \\
\hline Brown & 147 & 66.6 & 70 & 33.4 & & 126 & 57.1 & 91 & 42.9 & \\
\hline Black & 23 & 67.7 & 11 & 32.3 & & 26 & 73.8 & 8 & 26.2 & \\
\hline Economic class (ABEP) & & & & & $0.224^{1}$ & & & & & $0.587^{1}$ \\
\hline A-B & 102 & 74.9 & 35 & 25.1 & $0.132^{2}$ & 89 & 62.8 & 48 & 37.2 & $0.261^{2}$ \\
\hline $\mathrm{C}$ & 117 & 68.2 & 51 & 31.8 & & 95 & 55.8 & 73 & 44.2 & \\
\hline D-E & 16 & 60.9 & 11 & 39.1 & & 17 & 61.7 & 10 & 38.3 & \\
\hline School type & & & & & $0.216^{1}$ & & & & & $0.134^{1}$ \\
\hline Public & 162 & 68.5 & 74 & 31.5 & & 137 & 56.7 & 629 & 43.3 & \\
\hline Private & 73 & 75.1 & 23 & 24.9 & & 64 & 67.3 & 185 & 32.7 & \\
\hline
\end{tabular}

it continues

unadjusted $(\mathrm{OR}=2.30 ; 95 \% \mathrm{CI}=1.08-4.90)$ and adjusted $(\mathrm{OR}=2.32 ; 95 \% \mathrm{CI}=1.12-4.82)$ models (Table 4). Adolescents with swallowing disorders were $466 \%$ more likely to have Class III malocclusion $\quad(\mathrm{OR}=5.66 ; 95 \% \mathrm{CI}=1.35-23.71)$, $513 \%$ more likely to have posterior crossbite $(\mathrm{OR}=6.13 ; 95 \% \mathrm{CI}=2.76-13.62)$, and $353 \%$ more likely to have posterior open bite $(\mathrm{OR}=4.53$; $95 \% \mathrm{CI}=1.72-8.92)$ compared to those with normal swallowing (Table 4).

\section{Discussion}

This study suggests that malocclusion is not only an aesthetic problem but is probably associated with functional problems. Associations between breathing, phonation, chewing, and swallowing problems with malocclusion have been found, showing that the strength of association increases with increasing malocclusion severity, corroborating findings from other studies ${ }^{13,18}$. 
Table 1. Malocclusions and sociodemographic and behavioral characteristics of the sample, according to breathing and phonation alterations. São Luís, Brazil.

\begin{tabular}{|c|c|c|c|c|c|c|c|c|c|c|}
\hline \multirow{3}{*}{ Variables } & \multicolumn{4}{|c|}{ Breathing alterations } & \multirow{3}{*}{$\begin{array}{c}\mathrm{P}- \\
\text { value }\end{array}$} & \multicolumn{4}{|c|}{ Phonation alterations } & \multirow{3}{*}{$\begin{array}{c}\text { P- } \\
\text { value }\end{array}$} \\
\hline & \multicolumn{2}{|c|}{ No } & \multicolumn{2}{|c|}{ Yes } & & \multicolumn{2}{|c|}{ No } & \multicolumn{2}{|c|}{ Yes } & \\
\hline & $\mathbf{n}$ & $\%$ & $\mathbf{n}$ & $\%$ & & $\mathbf{n}$ & $\%$ & $\mathbf{n}$ & $\%$ & \\
\hline History of school failure & & & & & $0.913^{1}$ & & & & & $0.752^{1}$ \\
\hline No & 165 & 69.3 & 67 & 30.7 & & 144 & 59.5 & 88 & 40.5 & \\
\hline Yes & 70 & 70.3 & 30 & 29.7 & & 57 & 56.6 & 43 & 43.4 & \\
\hline Pacifier sucking & & & & & $0.986^{1}$ & & & & & $0.351^{1}$ \\
\hline No & 160 & 69.6 & 62 & 30.4 & & 134 & 56.1 & 88 & 43.9 & \\
\hline Yes & 75 & 69.7 & 35 & 30.3 & & 67 & 63.3 & 43 & 36.7 & \\
\hline Finger sucking & & & & & $0.398^{1}$ & & & & & $0.764^{1}$ \\
\hline No & 210 & 68.5 & 87 & 31.5 & & 181 & 58.0 & 116 & 42.0 & \\
\hline Yes & 25 & 77.4 & 10 & 22.6 & & 20 & 61.7 & 15 & 38.3 & \\
\hline Biting lips/cheeks & & & & & $0.476^{1}$ & & & & & $0.425^{1}$ \\
\hline No & 125 & 71.7 & 51 & 28.3 & & 108 & 60.7 & 68 & 39.3 & \\
\hline Yes & 110 & 67.3 & 46 & 32.7 & & 93 & 56.1 & 63 & 43.9 & \\
\hline Biting objects & & & & & $0.077^{1}$ & & & & & $0.510^{1}$ \\
\hline No & 154 & 73.8 & 52 & 26.2 & & 123 & 57.1 & 83 & 42.9 & \\
\hline Yes & 81 & 64.7 & 45 & 35.3 & & 78 & 60.1 & 48 & 39.9 & \\
\hline Nail biting & & & & & $0.978^{1}$ & & & & & $0.380^{1}$ \\
\hline No & 86 & 69.8 & 36 & 30.2 & & 75 & 62.2 & 47 & 37.8 & \\
\hline Yes & 149 & 69.6 & 61 & 30.4 & & 126 & 56.3 & 84 & 43.7 & \\
\hline Bruxism & & & & & $0.094^{1}$ & & & & & $0.121^{1}$ \\
\hline No & 193 & 72.9 & 74 & 27.1 & & 165 & 61.6 & 102 & 38.4 & \\
\hline Yes & 42 & 55.8 & 23 & 44.2 & & 36 & 45.2 & 29 & 54.8 & \\
\hline $\begin{array}{l}\text { Sleeping with hands under } \\
\text { the face }\end{array}$ & & & & & $0.252^{1}$ & & & & & $0.596^{1}$ \\
\hline No & 157 & 72.1 & 58 & 27.9 & & 130 & 59.5 & 85 & 40.5 & \\
\hline Yes & 78 & 64.9 & 39 & 35.1 & & 71 & 56.6 & 46 & 43.4 & \\
\hline Lingual interposition & & & & & $0.913^{1}$ & & & & & $0.249^{1}$ \\
\hline No & 201 & 69.9 & 86 & 30.1 & & 174 & 60.7 & 113 & 39.3 & \\
\hline Yes & 34 & 68.2 & 11 & 21.8 & & 27 & 46.7 & 18 & 53.3 & \\
\hline
\end{tabular}

Breathing and phonation were associated with malocclusion in anterior segments of mouth arches, measured by indicators such as DAI, which is aimed at the aesthetic evaluation of the anterior region ${ }^{4}$. The proper respiratory function (nasal breathing) requires labial sealing, and tongue must be in contact with the palate, allowing proper growth and craniofacial development $^{18}$. However, any respiratory disorder prevents labial sealing, and the tongue rests on the mouth floor, changing the palate shape, allowing the pressure of buccinator muscles to contribute to maxillary atresia and, therefore, to the onset of cross-sectional occlusal alterations ${ }^{18}$.
In the adjusted analysis for confounders, it was found that the likelihood of having defined, disabling, and Class III malocclusion was higher among patients with breathing problems, compared to those with normal breathing. This association may be due to the genetic pattern of the individual, and not by presenting Class III malocclusion since it shows a significant genetic causal factor. This relationship is still a controversial subject. While some authors ${ }^{11,33,34}$ found no significant association between mouth breathing and malocclusion and reported that the breathing mode does not influence craniofacial development, other studies ${ }^{10,35}$ established a 
Table 2. Malocclusions and sociodemographic and behavioral characteristics of the sample according to chewing and swallowing alterations. São Luís, Brazil.

\begin{tabular}{|c|c|c|c|c|c|c|c|c|c|c|}
\hline \multirow{3}{*}{ Variables } & \multicolumn{4}{|c|}{ Chewing alterations } & \multirow{3}{*}{$\begin{array}{c}\mathrm{P}- \\
\text { value }\end{array}$} & \multicolumn{4}{|c|}{ Swallowing alterations } & \multirow{3}{*}{$\begin{array}{c}\text { P- } \\
\text { value }\end{array}$} \\
\hline & \multicolumn{2}{|c|}{ No } & \multicolumn{2}{|c|}{ Yes } & & \multicolumn{2}{|c|}{ No } & \multicolumn{2}{|c|}{ Yes } & \\
\hline & $\mathbf{n}$ & $\%$ & $\mathbf{n}$ & $\%$ & & $\mathbf{n}$ & $\%$ & $\mathbf{n}$ & $\%$ & \\
\hline Malocclusion (DAI) & & & & & $0.554^{1}$ & & & & & $0.453^{1}$ \\
\hline Normal/Mild & 30 & 31.9 & 67 & 68.1 & $0.523^{2}$ & 74 & 80.0 & 23 & 20.0 & $0.160^{2}$ \\
\hline Defined & 16 & 21.6 & 66 & 78.4 & & 64 & 68.7 & 18 & 31.3 & \\
\hline Severe & 20 & 29.2 & 59 & 70.8 & & 57 & 73.6 & 22 & 26.3 & \\
\hline Disabling & 19 & 27.2 & 55 & 72.8 & & 50 & 67.7 & 24 & 32.3 & \\
\hline Malocclusion (Angle) & & & & & $0.012^{3}$ & & & & & $0.027^{3}$ \\
\hline Normal & 6 & 64.9 & 6 & 35.1 & & 12 & 100.0 & 0 & 0.0 & \\
\hline Class I & 41 & 20.3 & 157 & 79.7 & & 145 & 73.1 & 53 & 26.9 & \\
\hline Class II & 35 & 37.4 & 68 & 62.6 & & 78 & 77.6 & 25 & 22.4 & \\
\hline Class III & 3 & 11.9 & 16 & 88.1 & & 10 & 36.8 & 9 & 63.2 & \\
\hline Posterior crossbite & & & & & $0.031^{1}$ & & & & & $<0.001^{1}$ \\
\hline No & 74 & 30.7 & 203 & 69.3 & & 220 & 80.5 & 57 & 19.5 & \\
\hline Yes & 11 & 14.6 & 44 & 85.4 & & 25 & 40.3 & 30 & 59.7 & \\
\hline Posterior open bite & & & & & $0.225^{1}$ & & & & & $0.114^{1}$ \\
\hline No & 75 & 27.0 & 222 & 73.0 & & 228 & 75.6 & 69 & 24.4 & \\
\hline Yes & 10 & 35.0 & 25 & 65.0 & & 17 & 54.8 & 18 & 45.2 & \\
\hline Overbite & & & & & $0.078^{1}$ & & & & & $0.420^{1}$ \\
\hline No & 55 & 33.1 & 138 & 66.9 & & 147 & 75.5 & 46 & 24.5 & \\
\hline Yes & 30 & 18.2 & 109 & 81.8 & & 98 & 69.3 & 41 & 30.7 & \\
\hline Sex & & & & & $0.352^{1}$ & & & & & $0.712^{1}$ \\
\hline Female & 42 & 30.2 & 112 & 69.8 & & 117 & 74.9 & 37 & 25.1 & \\
\hline Male & 43 & 25.8 & 135 & 74.2 & & 128 & 72.0 & 50 & 28.0 & \\
\hline Race/color & & & & & $0.274^{1}$ & & & & & $0.190^{1}$ \\
\hline White & 25 & 32.8 & 56 & 67.2 & & 65 & 78.0 & 16 & 20.0 & \\
\hline Brown & 50 & 23.8 & 167 & 76.2 & & 153 & 69.8 & 64 & 30.2 & \\
\hline Black & 10 & 40.3 & 24 & 59.7 & & 27 & 80.2 & 7 & 19.8 & \\
\hline Economic class (ABEP) & & & & & $0.870^{1}$ & & & & & $0.231^{2}$ \\
\hline A-B & 40 & 29.6 & 97 & 70.4 & $0.218^{2}$ & 104 & 79.6 & 33 & 20.4 & $0.836^{2}$ \\
\hline $\mathrm{C}$ & 39 & 27.2 & 129 & 72.8 & & 118 & 68.1 & 50 & 31.9 & \\
\hline D-E & 6 & 25.9 & 21 & 74.1 & & 23 & 86.8 & 4 & 13.2 & \\
\hline School type & & & & & $0.758^{1}$ & & & & & $0.610^{1}$ \\
\hline Public & 56 & 27.2 & 710 & 72.8 & & 174 & 72.8 & 62 & 27.2 & \\
\hline Private & 29 & 31.0 & 220 & 69.0 & & 71 & 76.0 & 25 & 24.0 & \\
\hline History of school failure & & & & & $0.743^{1}$ & & & & & $0.825^{1}$ \\
\hline No & 61 & 27.1 & 171 & 72.9 & & 171 & 74.1 & 61 & 25.9 & \\
\hline Yes & 24 & 29.2 & 76 & 70.8 & & 74 & 71.9 & 26 & 28.1 & \\
\hline Pacifier sucking & & & & & $0.612^{1}$ & & & & & $0.816^{1}$ \\
\hline No & 54 & 26.9 & 168 & 73.1 & & 163 & 72.8 & 59 & 27.2 & \\
\hline Yes & 31 & 29.8 & 79 & 70.2 & & 82 & 74.5 & 28 & 25.5 & \\
\hline Finger sucking & & & & & $0.107^{1}$ & & & & & $0.188^{1}$ \\
\hline No & 70 & 25.8 & 227 & 74.2 & & 218 & 71.8 & 79 & 28.2 & \\
\hline Yes & 15 & 41.2 & 20 & 58.8 & & 27 & 83.9 & 8 & 16.1 & \\
\hline Biting lips/cheeks & & & & & $0.104^{1}$ & & & & & $0.821^{1}$ \\
\hline No & 48 & 31.6 & 128 & 68.4 & & 125 & 72.8 & 51 & 27.2 & \\
\hline Yes & 37 & 23.7 & 119 & 76.3 & & 120 & 74.0 & 36 & 26.0 & \\
\hline Biting objects & & & & & $0.251^{1}$ & & & & & $0.240^{1}$ \\
\hline No & 48 & 25.9 & 158 & 74.1 & & 155 & 76.2 & 51 & 23.8 & \\
\hline Yes & 37 & 30.1 & 89 & 69.9 & & 90 & 70.0 & 36 & 30.0 & \\
\hline
\end{tabular}


Table 2. Malocclusions and sociodemographic and behavioral characteristics of the sample according to chewing and swallowing alterations. São Luís, Brazil.

\begin{tabular}{|c|c|c|c|c|c|c|c|c|c|c|}
\hline \multirow{3}{*}{ Variables } & \multicolumn{4}{|c|}{ Chewing alterations } & \multirow{3}{*}{$\begin{array}{c}\text { P- } \\
\text { value }\end{array}$} & \multicolumn{4}{|c|}{ Swallowing alterations } & \multirow{3}{*}{$\begin{array}{c}\text { P- } \\
\text { value }\end{array}$} \\
\hline & \multicolumn{2}{|c|}{ No } & \multicolumn{2}{|c|}{ Yes } & & \multicolumn{2}{|c|}{ No } & \multicolumn{2}{|c|}{ Yes } & \\
\hline & n & $\%$ & n & $\%$ & & n & $\%$ & $\mathbf{n}$ & $\%$ & \\
\hline Nail biting & & & & & $0.153^{1}$ & & & & & $0.789^{1}$ \\
\hline No & 36 & 32.5 & 86 & 67.5 & & 90 & 74.6 & 55 & 25.4 & \\
\hline Yes & 49 & 25.1 & 161 & 74.9 & & 155 & 72.6 & 87 & 27.4 & \\
\hline Bruxism & & & & & $0.022^{1}$ & & & & & $0.284^{1}$ \\
\hline No & 75 & 31.0 & 192 & 69.0 & & 199 & 75.7 & 68 & 24.3 & \\
\hline Yes & 10 & 14.2 & 55 & 85.8 & & 46 & 63.1 & 19 & 36.9 & \\
\hline $\begin{array}{l}\text { Sleeping with hands } \\
\text { under the face }\end{array}$ & & & & & $0.442^{1}$ & & & & & $0.226^{1}$ \\
\hline No & 59 & 29.0 & 156 & 71.0 & & 153 & 70.7 & 62 & 29.3 & \\
\hline Yes & 26 & 25.5 & 91 & 74.5 & & 92 & 78.4 & 25 & 21.6 & \\
\hline Lingual interposition & & & & & $0.323^{1}$ & & & & & $0.699^{1}$ \\
\hline No & 70 & 25.7 & 217 & 74.3 & & 210 & 74.3 & 77 & 25.7 & \\
\hline Yes & 15 & 39.2 & 30 & 60.2 & & 35 & 68.4 & 10 & 31.6 & \\
\hline
\end{tabular}

${ }^{1}$ Chi-square test; ${ }^{2}$ Trend chi-square test; ${ }^{3}$ Fisher's exact test. Statistically significant P-values:P $<0.05$. DAI: Dental Aesthetic Index. ABEP: Economic class according to the Brazilian Association of Research Companies (Associação Brasileira de Empresas de Pesquisa - ABEP).

Source: Elaborated by the authors.

Table 3. Unadjusted and adjusted association between breathing and phonation alterations and malocclusions. São Luís, Brazil.

\begin{tabular}{|c|c|c|c|c|c|c|c|c|c|c|c|c|}
\hline \multirow{3}{*}{ Variables } & \multicolumn{6}{|c|}{ Breathing alterations } & \multicolumn{6}{|c|}{ Phonation alterations } \\
\hline & \multicolumn{3}{|c|}{ Unadjusted analysis } & \multicolumn{3}{|c|}{ Adjusted analysis } & \multicolumn{3}{|c|}{ Unadjusted analysis } & \multicolumn{3}{|c|}{ Adjusted analysis } \\
\hline & OR & $95 \% \mathrm{CI}$ & $\begin{array}{c}\mathrm{P}- \\
\text { value }\end{array}$ & OR & $95 \% \mathrm{CI}$ & $\begin{array}{c}\text { P- } \\
\text { value }\end{array}$ & OR & 95\%CI & $\begin{array}{c}P- \\
\text { value }\end{array}$ & OR & 95\%CI & $\begin{array}{c}\mathrm{P}- \\
\text { value }\end{array}$ \\
\hline \multicolumn{13}{|l|}{$\begin{array}{l}\text { Malocclusion } \\
\text { (DAI) }\end{array}$} \\
\hline Normal/Mild & 1 & - & - & 1 & - & - & 1 & - & - & 1 & - & - \\
\hline Defined & 4.31 & $1.44-12.92$ & 0.012 & $3.84^{1}$ & $1.45-10.12$ & 0.010 & 2.17 & $0.83-5.67$ & 0.107 & $2.01^{1}$ & $1.02-4.39$ & 0.047 \\
\hline Severe & 1.53 & $0.69-3.41$ & 0.273 & $1.44^{1}$ & $0.69-3.01$ & 0.303 & 1.44 & $0.65-3.21$ & 0.341 & $1.43^{1}$ & $0.62-3.28$ & 0.372 \\
\hline Disabling & 4.47 & $2.17-9.18$ & $<0.001$ & $4.34^{1}$ & $1.99-9.49$ & 0.001 & 3.03 & $1.56-5.91$ & 0.003 & $3.04^{1}$ & $1.55-5.96$ & 0.003 \\
\hline \multicolumn{13}{|l|}{$\begin{array}{l}\text { Malocclusion } \\
\text { according to Angle }\end{array}$} \\
\hline Normal/Class I & 1 & - & - & 1 & - & - & 1 & - & - & 1 & - & - \\
\hline Class II & 0.94 & $0.60-1.46$ & 0.763 & $0.87^{2}$ & $0.56-1.36$ & 0.528 & 1.69 & $1.01-2.85$ & 0.049 & $2.02^{2}$ & $1.28-3.18$ & 0.005 \\
\hline Class III & 3.45 & $0.67-17.72$ & 0.127 & $4.15^{2}$ & $1.19-14.54$ & 0.028 & 4.34 & $0.94-20.07$ & 0.059 & $3.07^{2}$ & $0.95-9.92$ & 0.059 \\
\hline
\end{tabular}

Posterior crossbite

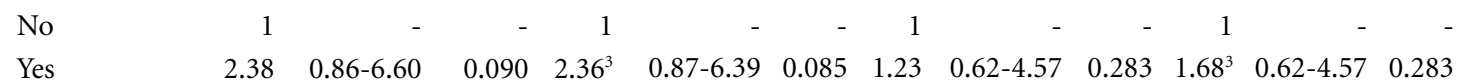

Posterior open

bite

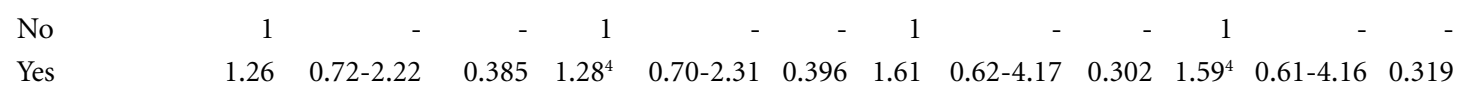

Overbite

$$
\begin{aligned}
& \begin{array}{llllllllllllll}
\text { No } & 1 & & - & - & 1 & - & - & 1 & - & - & 1 & - & -
\end{array} \\
& \begin{array}{lllllllllllll}
\text { Yes } & 1.53 & 0.82-2.85 & 0.166 & 1.64^{5} & 0.81-3.34 & 0.157 & 1.02 & 0.65-1.59 & 0.927 & 1.05^{5} & 0.66-1.68 & 0.821
\end{array}
\end{aligned}
$$

DAI: Dental Aesthetic Index. OR: Odds Ratio. 95\%CI: 95\% Confidence Interval. ${ }^{1}$ Odds ratio adjusted for school failure, bruxism and finger sucking habit; ${ }^{2}$ Odds ratio adjusted for sex, economic class, biting objects, finger sucking and lingual interposition; ${ }^{3}$ Odds ratio adjusted for economic class and habit of biting objects; ${ }^{4}$ Odds ratio adjusted for school failure; ${ }^{5}$ Odds ratio adjusted for school failure and economic class. 
Table 4. Unadjusted and adjusted association between chewing and swallowing alterations and malocclusions. São Luís, Brazil.

\begin{tabular}{|c|c|c|c|c|c|c|c|c|c|c|c|c|}
\hline \multirow{3}{*}{ Variables } & \multicolumn{6}{|c|}{ Chewing alterations } & \multicolumn{6}{|c|}{ Swallowing alterations } \\
\hline & \multicolumn{3}{|c|}{ Unadjusted analysis } & \multicolumn{3}{|c|}{ Adjusted analysis } & \multicolumn{3}{|c|}{ Unadjusted analysis } & \multicolumn{3}{|c|}{ Adjusted analysis } \\
\hline & OR & 95\%CI & $\begin{array}{c}\mathrm{P}- \\
\text { value }\end{array}$ & OR & 95\%CI & $\begin{array}{c}\text { p- } \\
\text { value }\end{array}$ & OR & $95 \% \mathrm{CI}$ & $\begin{array}{c}\mathrm{P}- \\
\text { value }\end{array}$ & OR & $95 \% \mathrm{CI}$ & $\begin{array}{c}\text { P- } \\
\text { value }\end{array}$ \\
\hline \multicolumn{13}{|l|}{ Malocclusion (DAI) } \\
\hline Normal/Mild & 1 & - & - & 1 & - & - & 1 & - & - & 1 & - & - \\
\hline Defined & 1.45 & $0.75-2.81$ & 0.247 & $1.39^{1}$ & $0.64-2.74$ & 0.415 & 0.90 & $0.45-1.82$ & 0.780 & $1.57^{1}$ & $0.61-4.06$ & 0.322 \\
\hline Severe & 1.16 & $0.46-2.92$ & 0.731 & $1.09^{1}$ & $0.43-2.74$ & 0.851 & 1.24 & $0.63-2.45$ & 0.532 & $1.38^{1}$ & $0.71-2.69$ & 0.322 \\
\hline Disabling & 1.38 & $0.49-3.87$ & 0.511 & $1.28^{1}$ & $0.51-3.21$ & 0.579 & 1.54 & $0.79-3.03$ & 0.207 & $1.94^{1}$ & $0.90-4.17$ & 0.087 \\
\hline \multicolumn{13}{|l|}{$\begin{array}{l}\text { Malocclusion } \\
\text { according to Angle }\end{array}$} \\
\hline Normal/Class I & 1 & - & - & 1 & - & - & 1 & - & - & 1 & - & - \\
\hline Class II & 0.77 & $0.31-1.96$ & 0.574 & $0.73^{2}$ & $0.30-1.77$ & 0.463 & 0.86 & $0.37-2.00$ & 0.715 & $0.80^{2}$ & $0.33-1.96$ & 0.612 \\
\hline Class III & 3.39 & $0.88-13.03$ & 0.072 & $3.66^{2}$ & $0.88-15.23$ & 0.072 & 5.13 & $1.11-18.78$ & 0.046 & $5.66^{2}$ & $1.35-23.71$ & 0.021 \\
\hline \multicolumn{13}{|l|}{ Posterior crossbite } \\
\hline No & 1 & - & - & 1 & - & - & 1 & - & - & 1 & - & - \\
\hline Yes & 2.30 & $1.08-4.90$ & 0.032 & $2.32^{3}$ & $1.12-4.82$ & 0.027 & 6.12 & $2.68-13.97$ & $<0.001$ & $6.13^{3}$ & $2.76-13.62$ & $<0.001$ \\
\hline \multicolumn{13}{|l|}{ Posterior open bite } \\
\hline No & 1 & - & - & 1 & - & - & 1 & - & - & 1 & - & - \\
\hline Yes & 0.60 & $0.24-1.49$ & 0.250 & $0.60^{4}$ & $0.24-1.51$ & 0.261 & 3.55 & $0.75-8.65$ & 0.124 & $4.53^{4}$ & $1.72-8.92$ & 0.037 \\
\hline \multicolumn{13}{|l|}{ Overbite } \\
\hline No & 1 & - & - & 1 & - & - & 1 & - & - & 1 & - & - \\
\hline Yes & 1.64 & $0.66-4.07$ & 0.265 & $1.79^{5}$ & $0.78-4.10$ & 0.157 & 1.36 & $0.61-3.04$ & 0.421 & $1.42^{5}$ & $0.59-3.42$ & 0.404 \\
\hline
\end{tabular}

DAI: Dental Aesthetic Index. OR: Odds Ratio. 95\%CI: 95\% Confidence Interval. ' ${ }^{1}$ Odds ratio adjusted for school failure, bruxism and finger sucking habit; ${ }^{2}$ Odds ratio adjusted for sex, economic class, biting objects, finger sucking and lingual interposition; ${ }^{3}$ Odds ratio adjusted for economic class and habit of biting objects; ${ }^{4}$ Odds ratio adjusted for school failure; ${ }^{5}$ Odds ratio adjusted for school failure and economic class.

Source: Elaborated by the authors.

direct relationship between mouth breathing and malocclusion.

In this research, the likelihood of having defined, disabling, and Class II malocclusion was higher among patients with phonation problems when compared to those with normal phonation. The strength of associations increased with increasing malocclusion severity. This result corroborates other studies ${ }^{13,36}$ that also found an association between phonation disorders and abnormal occlusion, including changes in the anterior segment of the mouth, such as anterior open bite.

Another research ${ }^{12}$ showed that Class III malocclusion, diastema, open bite, deep bite, and increased overjet tend to be associated with speech disorders, as these occlusal disorders modify phonemes. The most commonly found in literature regarding the relationship between speech with malocclusion as per Angle is the presence of lisping in individuals with Class II malocclusion, because the tongue tends to be positioned with high back and with its lower end in the oral cav- ity, favoring the change of the articulation point of fricative phonemes $/ \mathrm{s} /$ and $/ \mathrm{z} / /^{14}$.

The chewing and swallowing functions were associated with occlusal imbalances in the posterior region. Chewing disorders were associated with a higher prevalence of posterior crossbite among the adolescents included in our study. This association may due to posterior crossbites can produce a lack of contact in some teeth, thus, in spite of presenting all teeth, subjects with malocclusions may have less occlusal pairs, which can impair their mastication ${ }^{37,38}$.

Adolescents with swallowing disorders had a higher prevalence of Class III malocclusion, posterior crossbite, and posterior open bite when compared to those with normal swallowing. Another investigation ${ }^{39}$ found that tongue positioned in the genius region can determine mandibular prognathism, leading to Class III malocclusion. They also reported that if the tongue is positioned more forward and down, pressing the anterior teeth during swallowing leads to excessive pressure of the perioral muscles on flex- 
ible bone structure, resulting in a narrowing of the maxillary arch, supra-eruption of posterior teeth, buccoversion of anterior teeth, crossbite and open bite.

The results of the association between swallowing and malocclusion in this investigation also corroborate the study by Suliano et al. ${ }^{13}$, who found decreased normality of this function with increasing malocclusion severity. Atypical swallowing might lead to changes in the craniofacial pattern and mandibular morphology ${ }^{16}$.

A study ${ }^{29}$ with adolescents aged 12-15 years concluded that atypical swallowing had a significant impact on malocclusion, resulting in a higher frequency of crowding in anterior teeth, open bite, and spacing. Another research ${ }^{40}$ reported the importance of taking into consideration factors such as time, intensity, and frequency of habit to relate atypical swallowing as an etiological factor of malocclusion, which is challenging to evaluate in epidemiological studies. Marcomini et al. ${ }^{15}$ found an association between swallowing and malocclusion, specifically with open bite, but not with a posterior crossbite.

It is essential to know whether the "impulse" of the tongue during swallowing and other functional behavior is responsible, if it contributes to, or is a consequence of the development of malocclusion $^{17}$. The data obtained in this study do not allow establishing causal relationships. The cross-sectional design of this study does not allow saying whether the change in the function causes malocclusion or vice versa.

Some hypotheses are raised to explain such differences. Some authors studied malocclusion in the primary dentition in children aged 3-5 years $^{29}$, others in mixed dentition ${ }^{12}$, and others included adolescents aged 12 years in permanent dentition stage?. Our sample included only adolescents aged 12 years, considered as age-index for overall monitoring of various oral health problems by WHO for international comparisons and monitoring of trends ${ }^{1,2}$. Most studies include large age groups, which hampers the comparison of results since they can have deciduous, mixed, and permanent dentition in a single study.

Differences in sampling design may also have influenced the results because few studies have adopted the same design (probability cluster and stratified sampling, simple random selection) of this work. This type of method results in estimation with a lower probability of selection bias. Also, the use of multivariate analysis reduces the likelihood of confounding bias. An important aspect refers to the stomatognathic dysfunctions' diagnostic criteria. The tests considered different clinical aspects, objectively evaluated by previously trained examiners, according to the previous recommendations $s^{2,12,27,28}$, thus reducing the occurrence of measurement bias.

This study had some limitations, such as the time to complete the questionnaire and the fact that some students gave up participation during the examination, and some schools limit the time for the research so that students do not miss class. Nevertheless, these limitations do not detract from the usefulness of the study.

The strengths of this study are: $i)$ the probabilistic sampling design ensured good representativeness of the study population, and results can be extrapolated for a similar population and also for an external validation; ii) narrow confidence intervals that show the accuracy of association estimates; iii) malocclusion evaluation methods using different criteria - students were evaluated by Angle classification, DAI and other changes not included in DAI (posterior open bite, posterior crossbite, and overbite); iv) to the best of our knowledge, there are no previous studies considering these four oral functions alterations (breathing, phonations, chewing and swallowing); v) multivariate analyses contribute to the reduction of possible confounding biases; also, all analyzes considered deff and were weighted by the unequal selection probabilities of the subjects; vi) some strategies to reduce measurement bias were the use of examiners specialized in Orthodontics, that completed a training and a pilot study, with acceptable Kappa measurements.

However, it is necessary to develop longitudinal studies that follow people with malocclusion problems for a better understanding of the association with alterations of oral functions, as well as the dose-response relationship.

We emphasize the importance of this topic for public health because alterations in oral functions and severe malocclusions affect an important portion of the population, creating a demand for rehabilitation in different areas of health, especially Dentistry and Phonoaudiology.

\section{Conclusions}

Malocclusion is associated with functional alterations of the stomatognathic system. Breathing and phonation changes are associated with malocclusions in the anterior segments of the oral cavity, while chewing and swallowing are associated with malocclusions in the posterior segment 
of the oral cavity. The strength of the association between functional alterations and malocclusions increases with increasing malocclusion's severity. Therefore, the analysis of oral functions should be part of clinical examination protocols in health services.

\section{Collaborations}

HV Freitas and EBAF Thomaz worked on the conception of the work. HV Freitas and LFG Silva worked on the acquisition of data for the work. EBAF Thomaz worked on analysis and interpretation of data for the work. CMC Alves, LFG Silva, ALP Pereira, FN Hugo and EBAF Thomaz worked on drafting the work or revising it critically for important intellectual content; and final approval of the version to be published.

\section{Acknowledgments}

The authors are grateful to the Fundação de Amparo à Pesquisa e ao Desenvolvimento Científico e Tecnológico do Maranhão (FAPEMA) for supporting the translation of this article and for the scholarships; to the Coordenação de Aperfeiçoamento de Pessoal de Nível Superior - Brasil (CAPES) - finance code 001; and to the Conselho Nacional de Desenvolvimento Científico e Tecnológico ( $\mathrm{CNPq}$ ) for the research support grants.

\section{References}

1. Brasil. Ministério da Saúde (MS). Pesquisa Nacional de Saúde Bucal: resultados principais. SB Brasil, 2010. Brasília: MS; 2012.

2. World Health Organization (WHO). Oral Health Survey: basic methods. $5^{\text {th }}$ ed. Geneva: WHO; 2013.

3. Boronat-Catalá M, Bellot-Arcís C, Montiel-Company JM, Catalá-Pizarro M, Almerich-Silla JM. Orthodontic treatment need of 9, 12, and 15-year-old children according to the Index of Orthodontic Treatment and the Dental Aesthetic Index. J Orthod 2016; 43(2):130136.

4. Thomaz EB, Cangussu MC, Assis AM. Malocclusion and deleterious oral habits among adolescents in a developing area in northeastern Brazil. Braz Oral Res 2013; 27(1):62-69.

5. Nagarajappa R, Batra M, Sanadhya S, Daryani H, Ramesh G. Relationship between oral clinical conditions and daily performances among young adults in India - A cross-sectional study. J Epidemiol Glob Health 2015; 5:347-357.

6. Simões RC, Goettems ML, Schuch HS, Torriani DD, Demarco FF. Impact of malocclusion on oral health -related quality of life of 8-12 years old schoolchildren in Southern Brazil. Braz Dent J 2017; 28(1):105-112.

7. Narayanan RK, Jeseem MT, Kumar TVA. Prevalence of Malocclusion among 10-12-year-old Schoolchildren in Kozhikode District, Kerala: An Epidemiological Study. Int J Clin Pediatr Dent 2016; 9:50-55.

8. Uthaman C, Sequeira PS, Jain J, Shamarao S, Jain V. Perception of Personal Dental Appearance and Dental Aesthetic Index Score Among 18- to 20-year-old College Students in Rural South India. Oral Health Prev Dent 2015; 13:495-499.

9. Surtel A, Klepacz R, Wysokinska-Miszczuk J. The influence of breathing mode on the oral cavity. Pol Merkur Lekarski 2015; 39(234):405-407.

10. Retamoso LB, Knop LA, Guariza Filho O, Tanaka OM. Facial and dental alterations according to the breating pattern. J Appl Oral Sci 2011; 29(2):175-181.

11. Coelho ARDP, Tanaka O, Ribeiro JS, Machado MAN, Camargo ES. Transverse craniofacial dimensions in Angle Class II, Division1 malocclusion according to breathing mode. Braz Oral Res 2010; 24:70-75. 
12. Farronato G, Giannini L, Riva R, Galbiati G, Maspero C. Correlations between malocclusions and dyslalias. Eur J Paediatr Dent 2012; 13:13-18.

13. Suliano AA, Rodrigues MJ, Caldas Junior AF, Fonte PP, Porto-Carreiro PF. Prevalence of malocclusion and its association with functional alterations of the Stomatognathic system in schoolchildren. Cad Saude Publica 2007; 23(8):1913-1923.

14. Ferreira ML. The incidence of mouth breathing in subjects with class II occlusion. J Bras Fonoaudiol 1999; 1:83-96.

15. Marcomini L, Santamaria Jr M, Lucato AS, Santos JCB, Tubel CAM. Prevalence of malocclusion and its relationship with functional changes in the breathing and in the swallowing. Braz Dent Sci 2010; 13:52-58.

16. Fernandes LFT, Kochenborger R, Woitchunas FE, Woitchunas DR. The influence of atypical swallowing pattern in craniofacial and mandibular morphology. RFO 2010; 15:52-57.

17. Chiodelli L, Pacheco AB, Missau TS, Silva AMT, Corrêa ECR. Association among Stomatognathic functions, dental occlusion, and temporomandibular disorders signs in asymptomatic women. Rev CEFAC 2015; 17(1):117-125.

18. Suliano AA, Borba PC, Rodrigues MJ, Caldas Júnior AF, Santos FAV. Prevalence of malocclusion and functional changes among students by the Family Health Program in Juazeiro do Norte, Ceará, Brazil. $R$ Dental Press Ortodon Ortop Facial 2005; 10:103-110.

19. Malta M, Cardoso LO, Bastos FI, Magnanini MMF, Silva CMFP. STROBE initiative: guidelines on reporting observational studies. Rev Saude Publica 2010; 44(3):559-565.

20. Programa das Nações Unidas para o Desenvolvimento (PNUD). Atlas do Desenvolvimento Humano no Brasil 2013. Ranking. Todos os Estados. 2010 [Internet]. [cited 2018 May 10]. Available from: http://atlasbrasil. org.br/2013/pt/ranking.

21. Emmerich A, Fonseca L, Elias AM, Medeiros UV. The relationship between oral habits, oronasopharyngeal alterations, and malocclusion in preschool children in Vitória, Espírito Santos, Brazil. Cad Saude Publica 2004; 20(3):689-697.

22. Thomaz EBAF, Cangussu MCT, Silva AAM, Assis AMO. Is Malnutrition Associated with Crowding in Permanent Dentition? Int J Environ Res Public Health 2010; 7:3531-3544.

23. Thomaz EBAF, Cangussu MCT, Assis AMO. Maternal breastfeeding parafunctional oral habits and malocclusion in adolescents: A multivariate analysis. Int $J$ Pediatr Othorhinolaryngol 2012; 76(4):500-506.

24. Santos PCF, Monteiro ALB, Rocha RG, Chaves Júnior CM. An alternative tool for assessing the Dental Aesthetic Index. Rev Clin Ortodon Dental Press 2008; 7:34-39.

25. Katz MI. Angle classification revisited. 1: Is current use reliable? Am J Orthod Dentofacial Orthop 1992; 102(2):173-179.

26. Jenny J, Cons NC. Establishing malocclusion severity levels on the Dental Aesthetic Index (DAI) scale. Aust Dent J 1996; 41(1):43-46.

27. Borzabadi-Farahani A, Eslamipour F, Asgari I. A comparison of two orthodontic aesthetic indices. Aust Orthod J 2012; 28(1):30-36.
28. Miyawaki S, Tanimoto Y, Araki Y, Katayama A, Kuboki T, Takano-Yamamoto T. Movement of the lateral and medial poles of the working condyle during mastication in patients with unilateral posterior crossbite. Am J Orthod Dentofacial Orthop 2004; 126(5):549-554.

29. Souza JF, Grechi TH, Anselmo-Lima WT, Trawitzki LVV, Valera FCP. Mastication and deglutition changes in children with tonsillar hypertrophy. Braz J Otorhinolaryngol 2013; 79(4):424-428.

30. Fuchs SC, Victora CG, Fachel J. Hierarchical model: a proposal for model to be applied in the investigation of risk factors for dehydrating diarrhea. Rev Saude Publica 1996; 30(2):168-178.

31. Rothman KJ, Greenland S, Lash TL. Epidemiologia moderna. $3^{\text {rd }}$ ed. Porto Alegre: Artmed; 2011.

32. D'Onofrio L. Oral dysfunction as a cause of malocclusion. Orthod Craniofac Res 2019; 22(1):43-48.

33. Baldrighi SEZM, Pinzan A, Zwicker CVD, Michelini CR, Barros DR, Elias F. The importance of breastfeeding in preventing myofunctional and orthodontic changes. $R$ Dental Press Ortodon Ortop Facial 2001; 6:111-121.

34. Occasi F, Perri L, Saccucci M, Di Carlo G, Ierardo G, Luzzi V, Castro G, Brindisi G, Loffredo L, Duse M, Polimeni A, Zicari AM. Malocclusion and rhinitis in children: an easy-going relationship or a yet to be resolved paradox? A systematic literature revision. Ital J Pediatr 2018; 44(1):100.

35. Fraga WS, Seixas VM, Santos JC, Paranhos LR, César CP. Mouth breathing in children and its impact in dental malocclusion: a systematic review of observational studies. Minerva Stomatol 2018; 67(3):129-138.

36. Ocampo-Parra A, Escobar-Toro B, Sierra-Alzate V, Rueda ZV, Lema MC. Prevalence of dyslalias in 8 to 16 year-old students with anterior open bite in the municipality of Envigado, Colombia. BMC Oral Health 2015; 15:77.

37. Bae J, Son WS, Kim SS, Park SB, Kim, YI. Comparison of masticatory efficiency according to Angle's classification of malocclusion. Korean J Orthod 2017; 47(3):151-157.

38. Choi TH, Kim BI, Chung CJ, Kim HJ, Baik HS, Park YC, Lee KJ. Assessment of masticatory function in patients with non-sagittal occlusal discrepancies. J Oral Rehabil 2015; 42(1):2-9.

39. Serra-Negra JMC, Por Deus IA, Rocha Jr JF. Study of the association between breastfeeding, oral habits and malocclusion. Rev Odontol USP 1997; 11:79-86.

40. Vieira MD, Vilella OV. Cephalometric evaluation of the oropharyngeal space in patients with atypical swallowing. Rev Odonto Cien 2008; 23:26-30.

Article submitted 15/05/2019

Approved 24/04/2020

Final version submitted 26/04/2020

Chief editors: Romeu Gomes, Antônio Augusto Moura da Silva 